\title{
Assessing the Importance of Data Factors of Data Quality Model in the Business Intelligence Area
}

\author{
Aylin Hejazi, Neda Abdolvand, and Saeedeh Rajaee Harandi
}

\begin{abstract}
Business Intelligence (BI) is defined as the intelligent transfer of data from all variety of sources with a view to providing a real time quantification of business performance. Moreover, by utilizing BI senior managers are able to make better-informed decisions, guided by the integration of comprehensive information from all facets of an organization in real time. Further, the quality of corporate decision making is directly dependent upon the quality of data. Quality dimensions have proven to be of importance in evaluating how an organization understands and manages its data. Hence, in this study we employed the data quality model of Wang and Strong as our benchmark, and by questioning experts five additional factors (Usefulness, Efficiency, Availability, Navigation, and Usability) were added to the model to improve the reference model. Furthermore, the importance of these factors (and how they relate to each other, in terms of their dimensions) will be measured by the AHP method and an expert panel. Finally, we will reveal how the findings demonstrate that the intrinsic, contextual, representational and accessibility IQs in terms of their dimensions and reputation, accuracy, believability, and reliability, proved to be the most important factors..
\end{abstract}

Index Terms-Business intelligence, data, data quality, data quality model.

\section{INTRODUCTION}

Historically, business intelligence appeared in the mid-1960s within management information systems. Moreover, with the rapid evolution of computer technology in the 1970s decision support systems subsequently emerged [1] The extant technology continued to improve until the advent of executive information and data warehouse systems. However, the subsequent massive growth in demand for complex data warehouse systems prompted researchers to use a new term of reference, namely BI. Since that time, BI systems have become the predominant technology utilized by organizations to improve their performance and quality of information in support of decision-making processes [2]. In fact, BI has emerged as the leading solutions for the improvement of the decision-making process [3]. BI also serves as a working map for companies to maximize their profitability through a comprehensive data analysis, and its wider availability will enable companies to benchmark their performance relative to those of other organizations [4]. In case of appropriate implementation of BI, its massive implementation costs would be profitable [5]. BI has been

Manuscript received November 23, 2016; revised February 28, 2017.

The authors are with the Faculty of Social Science and Economics, Alzahra University, Tehran, Iran (email: aylinhejazi@gmail.com, abdolvand@gmail.com, saeedeh.rh@gmail.com). successfully used to augment a variety of programs, including selling and marketing analysis, planning and forecasting, financial strengthening, legal reporting, and budget analysis [6]. According to the studies, appropriate implementation and use of BI can result in numerous benefits, including profit increase, cost reduction, and efficiency improvement. Although a lot of organizations implement BI to realize its benefits, not all of these efforts will be successful [7]. In addition, the projects of implementing BI systems are very expensive, and yet, according to studies, the probability of failure of these projects still remains high. Findings indicate that only $24 \%$ of the BI projects are identified as very successful projects [8]. Furthermore, according to the studies, the cost of implementing BI will vary from 50 thousand dollars to billions of dollars for implementing complex Data Warehouse projects. Hence, a large amount of money is spent by the companies on BI projects to establish these systems, without guarantee of achieving any success or positive impact [9]-[11]. Implementation of BI systems is intricate, and uses a significant number of sources [12]. However, if the organizations can implement it successfully, it will provide technical solutions with extensive analytical capabilities that are presented to the stakeholders at different levels of the organization, which will help the people to acquire useful information for effective decision making [13]. Undoubtedly, timely and accurate business decisions need the right information at the right time which is the prerequisite for making reliable, correct and accurate business decisions [14]. Low-quality data can have a significant social and economic impact. Thus, it is essential to understand the data quality (DQ) from the consumer's perspective in order to improve its quality. Discovering the impact of quick and timely access to information in regard to business decisions is not easy [15]. Similarly, finding the impact of timely and quick access to information for making business decisions is not easy achievable, and measuring and assessing its impact on general business is even more difficult [16]. Therefore, Understanding and managing data by organizations in order to create procedures to ensure data quality is important [17]. Moreover, whereas any problem in data quality will affect other dimensions, identifying data quality dimensions is a vital step in measuring data quality [18]. Hence, the question arises, how important are the factors of data quality and dimensions to each other in assessing data quality? This study employed the data quality model by [15], which is one of the essential data quality frameworks to have analyzed data quality from the perspective of data consumers [19]. They believed data consumers have a broader understanding of data quality in comparison to information systems specialists. Since this model was introduced in 1996, other effective 
factors have also been identified in the following years. Hence, in this study, other factors were added into the model using an expert panel, and a conceptual model was developed. Then, by paired comparison analysis and the AHP method the importance of each factor and data dimension in Iranian organizations, where the information systems are older and less ready to implement these systems, were measured. For this purpose, the research literature was reviewed first and factors and variables were extracted. Then the research methodology is reviewed and research model is developed. The conclusion and recommendation of the study are discussed in the last part.

\section{LITERATURE REVIEW}

$\mathrm{BI}$ is a managerial approach that allows an organization to realize useful and relevant information for decision-making. BI helps organizations to make real-time and quick decisions through data, and it prevents a great number of problems and errors [20], [21]. Thus, BI solutions offer the ability to extract, cleans and aggregate data from multiple operational systems in a single data warehouse [21]. These solutions enable organizations to improve decision-making and require processes, skills, technology and data [20]. BI systems combine data gathering and storage and knowledge management with analytical tools so that they offer complex and competitive information for decision-makers [22]. From this definition, the importance of data in BI systems is apparent. Technical capabilities and data analysis applications are the requirements of eliciting the value of BI and supporting it [23]. Different data-driven applications, such as data warehousing use different data quality software tools to improve the business processes [26]. Data warehouses are the central elements of BI, which integrate data from different transmission information systems with the aim of fulfilling analytical purposes [24], [25]. Data quality is a multidimensional concept and has different characteristics; it is possible that data used in a particular situation will not be useful for other situations and applications. In general, data quality is defined as data that is suitable for use [15]-[27]. Data are directly related to BI productivity; this means that not only should the right information reach the right user at the right time but also that data should be delivered for specific users in the best way in order to customize it to maximize the BI profit [27]. Therefore, it is important for organizations to understand and manage data in order to create procedures to ensure data quality [17]. Information quality is also another complex and multifaceted issue that is influenced by three factors namely user's understanding, information, and the process of accessing information [27].

The rapid growth of the Internet as an environment for information exchange and the lack of standards in this area for applying information cause problems for data and information in terms of quality [29]. Data quality problems have expanded in data warehouses and implementations of enterprise resource planning systems. Low-quality data can have a significant social and economic impact. Data quality management is an important area for studies and investment in information technology [18]. Real assessment of data quality dimensions is difficult due to the abstractness of the quality concept [29]. A great number of studies have been conducted within the field of data quality. For example, Reference [30] presented advices for improving data quality. Reference [31] reviewed all data and information proposed models between 1996 and 2002 to determine the quality factors, and finally presented the IQIP model as an approach to managing the implementation of the Internet crawling search engine algorithms related to quality [31]. Reference [15] provided a conceptual framework with four categories and fifteen dimensions that assessed data quality from the customer's perspective. Reference [17] in their study examined the cultural and social dimensions of data quality, and indicated that creating a common understanding and awareness of the biases of vital data quality factors at the social level are very vital. They also suggested that perspective analysis, conflicts solving, and consideration of culture are important strategies for improving data quality at the social level. Reference [31] provided a conceptual framework to assess the quality of five information systems along five dimensions: presentation quality, text quality, transaction quality, access quality, and ergonomic quality. They also showed that the quality criteria are not developed in separate processes of creating data and data usage fields [32]. Furthermore, [18] identified the dimensions used regularly, and then mapped the data problems to the identify dimensions, showing the need for standardization in this area. Although models for assessing DQ exist, DQ models in the field of metadata are scarce, however, to bridge this gap in the literature, [29] provided a classification based on user's assessment or on information quality criteria, identifying three sources of metadata information quality: user, source, and query process. Based on ISO25012 and ISO25024, [33] created the 3C model consisting of three DQ dimensions, namely contextual consistency, operational consistency, and temporal consistency, which are used to assess the quality of data used in a metadata set. In another, [34] presented the 3A model, which includes three DQ characteristics used to assess data quality level in huge projects: contextual adequacy, temporal adequacy, and operational adequacy. Further, they showed that this model can be an appropriate method for achieving the level of input DQ in big data analysis in order to enhance reliability and accuracy. Reference [35] developed the two-stage model for managing the quality of SMCPS's data and demonstrated that the two-stage control strategy is better than one-stage or random control for ensuring DQ and saving control costs. Moreover, local data were produced at lower control cost and with better quality. Reference [36] conducted a model with five quality dimensions, to demonstrate the usefulness of the connected data and assess the quality two sets of related data. Moreover, they showed that amount of data, concise, completeness, navigability, and interlinking are effective factors in assessing connected databases. Reference [36] reviewed data quality frameworks proposed over the past ten years and identified two pragmatic and analytic criteria with four common factors namely objectivity, accessibility, conciseness, and accuracy in the reviewed frameworks which show the importance of these factors for information quality. Reference [37] presented a PSP/IQ conceptual model based on reliability, usability, usefulness, and correct information to 
assess how correct information is developed and how organizations can provide usable and reliable information services to their customers. Reference [38] developed a theoretical model to study the factors that affect users' detection of World Wide Web information quality problems and identified fourteen dimensions and nineteen indicators related to information quality problems. Reference [39] described an approach that combines similarity rankings with quality rankings in centralized and distributed environments; they also identified six quality criteria: generality, accessibility, availability, power, popularity, and consistency, and it suggested that combining quality criteria can improve effectiveness in both centralized and distributed environments In another study, [40] indicated that completeness, accuracy, consistency, timeliness, and correctness are essential aspects of data quality. at the same study Reference [41] indicated the positive effects of Completeness, Accuracy, Correctness, Consistency, Timeliness on data quality Reference [42] indicated that Six quality metrics, including the currency, availability, information to noise ratio, authority, popularity, and cohesiveness are effective on the search effectiveness in Centralized/Distributed Information Retrieval on the World Wide Web. Reference [43] have identified the Decision-maker quality; Data quality of the BD sources; Staff; Flexible infrastructure; Routinizing and standardization; Process integration and standardization; Collaboration; Knowledge exchange; BDA capabilities; Relational governance; Contractual governance as the effective factors on the big data decision-making quality. Moreover, Reference [44] used a combinatory approach of subjective and objective to assess data quality and suggested that data quality assessment requires the awareness of the principles of developing subjective and objective data quality metrics.

Research carried out about data quality and their dimensions are summarized in Table I.

TABLE I: DATA QUALITY CATEGORIZATION IN THE LITERATURE

\begin{tabular}{ccc}
\hline \hline Source & Target & Dimensions \\
\hline [34] & Data Quality Model For Assessing & Contextual Adequacy, Temporal Adequacy, Operational Adequacy \\
& Big Data Quality & Subject Criteria : Believability, Concise representation, Interpretability, Relevancy, Reputation, \\
& & Understandability, Value-Added
\end{tabular}

Prioritization of Dimensions, Skills And Data Quality Requirements

Assessing the Effective Factors on Big Data Decision-Making Quality

Framework For Assessing Information System Quality

Improvement of Data Quality

To PROVIDE a Conceptual Framework For Information Quality in the Website Context

To Conduct a Conceptual Model For Checking Data Quality

Mapping Information Quality Dimension Into The Psp/Iq Model

To provide a Framework For Checking Cultural And Social Dimensions of Data Quality

To Propose A Theoretical Model To Find Effecting Factors of World Wide Web Information Quality Problems
Accuracy: Accuracy, Unbiased, Believability, Traceability; Accessibility: Accessibility, Believability, Appropriate Amount of Information; Usefulness: Interpretability, Understandability, Ease of Manipulation, Consistent Representation, Value Added; Relevance: Relevant, Concise Representation, Up To Date, Reputation, Value Added; Security: Security, Traceability

Decision-maker quality; Data quality of the BD sources; Staff; Flexible infrastructure; Routinizing and standardization; Process integration and standardization; Collaboration; Knowledge exchange; BDA capabilities; Relational governance; Contractual governance

Ergonomic Quality : Ease of Navigation, Conformability, Trainability, Visual signals, Audio signals Accessibility Quality: Technical access, System availability, Technical security, Data accessibility, Data sharing, Data convertibility Transactional Quality : Controllability, Error tolerance, Adaptability, System feedback, Efficiency, Responsiveness Contextual Quality : Value added, Relevancy, Timeliness, Completeness, Appropriate data Representation Quality : Interpretability, Consistency, Conciseness, Structure, Readability, Contrast

Intrinsic: Accuracy, Lineage, Semantic, Structure

Contextual: completeness, consistency, currency, timeliness, reasonableness, and identifiability Qualitative dimensions

Content Quality : Relevant Information, Sound Information

Media Quality : Optimized Process, Reliable Infrastructure

Intrinsic IQ : Accuracy, Objectivity, Believability, Reputation

Accessibility IQ : Accessibility, Security

Contextual IQ : Relevancy, Value-Added, Timeliness,

Completeness, Amount of Info

Representational IQ : Interpretability, Ease of Understanding, Concise Representation, Consistent Representation

Product Quality : Sound Information, Useful Information

Service Quality : Dependable Information, Useable Information

Syntactic : Consistent

Semantic : Complete and Accurate

Pragmatic : Usable and Useful

Social : Shared understanding of meaning

Accuracy, Completeness, Relevance, Timeliness, Amount of Data 


\begin{tabular}{|c|c|c|}
\hline Source & Target & Dimensions \\
\hline [41] & $\begin{array}{l}\text { Combination Of Quality Criteria in } \\
\text { Recovering } \\
\text { Distributed/Centralized } \\
\text { Information In World } \\
\text { Wide Web }\end{array}$ & Currency, availability, information-to-noise ratio, authority, popularity, cohesiveness \\
\hline [31] & $\begin{array}{l}\text { To Conduct a Conceptual Model For } \\
\text { Checking Information } \\
\text { Quality in World Wide } \\
\text { Web }\end{array}$ & Identify, Quantify, Implement and Perfect. \\
\hline [42] & $\begin{array}{l}\text { Reviewing Data Quality Anthology } \\
\text { in Managing Chronic } \\
\text { Diseases }\end{array}$ & Completeness, Accuracy, Correctness, Consistency, Timeliness \\
\hline [44] & To Assess The Data Quality & $\begin{array}{l}\text { Value Added, Understandability, Timeliness, Security, Reputation, Relevancy, Objectivity, } \\
\text { Interpretability, Free of Error, Ease of Manipulation, Consistent Representation, Concise } \\
\text { Representation, Completeness, Believability, Appropriate Amount of Data, Accessibility }\end{array}$ \\
\hline
\end{tabular}

According to studies, a lot of quality models have been developed that some of them check the quality indicators, and some of them check the quality dimensions and categorized data and information according to visions and applications, and the number of levels and factors were different and in some cases there were overlaps. Moreover, methods were different and some of them developed a model based on literature review, and some of them were case studies. A series of features were same on most of the models. In addition, based on organizations need the focus of the model is different, and considers special cases. Moreover, according to findings, few studies have been done in this field in Iran. Hence, it is necessary for organizations to have a comprehensive model to identify the most common and effective dimensions in data quality. Therefore, this study reviewed 41 articles related to data, information, and data models using content analysis and comprehensive study of literature. Among the different data models that have been checked, data quality model of [15] with four dimensions and fifteen indicators, is still one of the most important frameworks [19]. Moreover, this framework is one of the few studies that focus on the concept of data quality from the customer's perspective, and that was chosen as the reference model. Another reason for choosing this model is that this model has most overlapping indicators with the twenty common indicators which are introduced by [31] due to reviewing all data or information quality models from 1996 until 2002. It is clear that other indicators have been proposed since 1996. Therefore, six other indicators namely, usefulness, usability, availability, navigation, and reliability were added to this model using expert's opinions, and classified in their right dimensions. Hence, conceptual model of study was developed, and was identified the most important factors of assessing data quality.

\section{CONCEPTUAL FRAMEWORK}

The aim of this study is to identify the most important factors of data quality. The question that arises from the basis of the research is that how important are each indicators in their dimensions and each dimensions toward each other? In order to answer this question, this study consists of two phases; in the first stage, by using content analysis and comprehensive literature study data quality model of [15] which examine data quality from customer's perspective, has been considered as a reference model, and by using expert panel six indicators of usefulness, usability, availability, efficiency, navigation, and reliability added to the model, and the conceptual model was developed. Then, academic experts requested to categorize these six indicators into model's dimensions. After finalization of indicator's categorization in model's dimensions, the validity of the proposed dimensions for each indicator was confirmed by using CVR. In the second stage, by using an analytic hierarchy process (AHP) was requested from academic experts to score the importance of proposed indicators in their dimensions and the importance of each dimension based on AHP method. Therefore, indicators and dimensions were ranked using expert panel. The final model has 21 indicators and four dimensions namely Intrinsic DQ which includes features and criteria that emphasize quality must be in data's nature, Accessibility DQ which is determined that data quality requirement should be considered in the context of the tasks at hand, Contextual DQ, and Representational DQ both emphasize the importance of the role of systems, and Contextual DQ were considered important by information systems professionals.

Representational DQ same as Accessibility DQ underlined the importance of the role of systems [15] (Fig. 1).

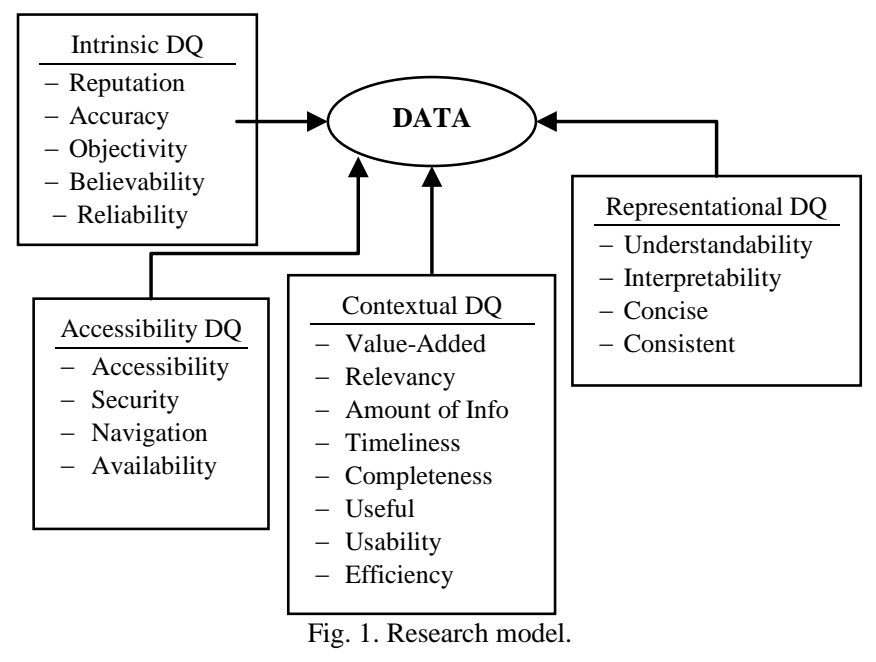

\section{HYPOTHESIS DEVELOPMENT}

In this study, two questionnaires were used to validate model variables and to survey expert's opinions. The first questionnaire includes questions to measure adding of six indicators to the reference data quality model by [15]. From 37 questionnaires distributed among academic and industry 
experts and executives, 26 questionnaires were received. The experts agreed with each factor based on five-point Likert's scale ranging from insignificant (1) to very important (5). The validity of the proposed dimension was calculated by CVR index. When more than half of the expert panel chooses the important option, CVR is between 0 to $99 \%$, and the CVR is negative otherwise. When all the experts chose the important option, CVR is 1 ; to make it easier this amount is adjusted to 0.99 . To approve the indicators in their proposed dimensions, scores that were obtained from this formula must be above 42.0. Based on the results in Table II all indicators were accepted in the proposed dimensions.

In the next step, AHP questionnaire is used to determine the importance of the proposed indicators in their dimensions and the importance of each dimensions itself. AHP questionnaire composed of five matrix which four of them related to indicator's dimensions namely Intrinsic DQ (Reputation, Accuracy, Objectivity, Believability, Reliability) and Representational DQ (Understandability, Interpretability, Concise, Consistent) and Accessibility DQ (Accessibility, Security, Navigation, Availability) and Contextual DQ (Value-Added, Relevancy, Amount of Info, Timeliness, Completeness, Useful, Usability, Efficiency). The last matrix is to compare the importance of each dimension in comparison to each other. From 37 distributed AHP questionnaires among academic and industry experts and executives, only 10 questionnaires were returned.

TABLE II: CVR RESULTS

\begin{tabular}{cccccccc}
\hline \hline Dimension & & Contextual DQ & & \multicolumn{2}{c}{ Accessibility DQ } & Intrinsic DQ \\
\hline Indicator & Efficiency & Usability & Usefulness & Navigation & Availability & Reliability \\
\hline CVR & 0.6 & 0.52 & 0.76 & 0.84 & 1 & 0.84 \\
\hline \hline
\end{tabular}

TABLE III: DIMENSIONS AND INDICATORS IN ORDER OF IMPORTANCE

\begin{tabular}{|c|c|c|c|}
\hline Dimensions in order of Importance & Mean Rank & Indicators in order of Importance & Mean Rank \\
\hline \multirow[t]{5}{*}{ Intrinsic DQ } & \multirow[t]{5}{*}{0.387} & Reputation & 0.244 \\
\hline & & Accuracy & 0.208 \\
\hline & & Reliability & 0.199 \\
\hline & & Believability & 0.179 \\
\hline & & Objectivity & 0.170 \\
\hline \multirow[t]{8}{*}{ Contextual DQ } & \multirow[t]{8}{*}{0.265} & Value-Added & 0.181 \\
\hline & & Timeliness & 0.174 \\
\hline & & Relevancy & 0.170 \\
\hline & & Amount of Info & 0.109 \\
\hline & & Usability & 0.101 \\
\hline & & Useful & 0.098 \\
\hline & & Completeness & 0.084 \\
\hline & & Efficiency & 0.083 \\
\hline \multirow[t]{4}{*}{ Accessibility DQ } & \multirow[t]{4}{*}{0.197} & Security & 0.391 \\
\hline & & Accessibility & 0.295 \\
\hline & & Availability & 0.195 \\
\hline & & Navigation & 0.119 \\
\hline \multirow[t]{4}{*}{ Representational DQ } & \multirow[t]{4}{*}{0.191} & Understandability & 0.374 \\
\hline & & Consistent & 0.352 \\
\hline & & Concise & 0.144 \\
\hline & & Interpretability & 0.130 \\
\hline
\end{tabular}

\section{RESEARCH Methodology}

In this study, AHP is used to assess the importance of indicators in their dimensions and eventually the importance of dimensions toward each other. AHP is a systematic approach that is designed for non-structural problems to help decision-makers, and this is achieved by dividing the problem into a series of criteria and options. Then, the problems assess by Paired Comparison of criteria and options in this hierarchy. In addition, the AHP assesses the compatibility of paired comparison, which is created during hierarchy. Moreover, this process provides the ability to prioritize the criteria and alternatives. The first stage of this procedure is to list the criteria and options. After that, the participants are requested to score paired comparison between elements of hierarchy by using AHP method, which are equal importance (1), moderate importance (3), strong importance (5), very strong importance (7), and extreme importance (9) [45]. The geometric mean is used to combine the paired comparison. Then, to normalize the geometric mean of each row, it should be divided by the sum of the geometric mean columns. After assigning a weight for each criterion, options should be compared in pairs based on each criterion. Finally, each option is calculated by the total of the product of choice priority based on "I* priority criterion" criteria. After the finalization of the classification of the indicator's proposed dimensions, dimensions and indicators have been analyzed by the expert choice software, and the questionnaire incompatibility factor was assessed. If the calculated number for inconsistency is less than 0.1 , there will be essential agreement; otherwise, experts should reconsider their responses. According to the results of the incompatibility factor, all factors were less than 0.1 and acceptable. According to a poll of experts who was conducted, four dimensions of data quality are named; intrinsic $D Q$, contextual DQ, accessibility DQ, and representational DQ, in order of their importance. These dimensions and their indicators are shown in Table III according to their 
importance.

\section{CONCLUSION AND RECOMMENDATIONS}

With the development of science and technology, the challenges have changed, and unlike in the past, obtaining information is not the only concern, Nowadays, due to the proliferation of information, accessing the needed information within the critical time is important. BI is a method that can solve this problem, and it can provide reports for senior managers. As a result, successful and measured implementation is essential. Therefore, in this study, the data quality model of [15] is used with an aim of comparing the importance of data dimensions' indicators and dimensions themselves, and the 20 common indicators that were introduced by [30] were combined with the 15 indicators of the [15] data quality model. By using an expert panel, the importance of the indicators is examined. According to AHP results, reputation, accuracy, and reliability are the most essential indicators which are compatible with results of [31] and [15]. According to data analysis findings, using AHP method revealed that among the four introduced dimensions, intrinsic DQ is the most important dimension, and four out of five indicators of this dimension are scored the highest importance among all indicators. In addition, another intrinsic DQ indicator namely, objectivity after value-added from contextual DQ is the sixth important indicator. In the next place, timeliness and relevancy are belonged to the second important dimension namely contextual DQ. The next priority is the security of accessibility DQ, and finally, there are representational DQ's indicators. Similarly, indicator's rankings are fully matched with dimension's importance, and the results are acknowledged. Based on results, clearly data intrinsically should have some features so that it can be displayed, or make it available, or to provide contextual data quality. In the following, data quality indicators related to accessibility and ultimately accessibility and representational data quality are located. For instance, data with the initial conditions should exist so that they can become consistent and concise in the future, or they can be interpreted with data collection. According to the results, although companies improve data quality of the tools and practical approaches, efforts should be focused on accuracy, and they should use better control procedures to ensure the accuracy of data.

With the development of technology and information, it is undeniable that other indicators will be added to data variable. Even the existence of some indicators may need to be revised. To improve data quality, precisely what the data quality means for the data consumers should be understood first [15]. With the development of organizations and technology, the importance of these dimensions will change. The accuracy in the research of [30] also is the most repetitive indicator. With regard to the importance of these 21 indicators, companies can easily determine which indicators they should give special care and attention to, and on which indicators they need to spend more money and time. Therefore, organizations should implement and improve their BI systems in such a way that they comply with the percentage of importance pertaining to that indicator or dimension. Generally, the intrinsic data quality dimension should be considered more than other dimensions. By way of illustration, the data source from which are data obtained, should be reliable, thus ensure the accuracy of data. If data are collected and created properly, the amount spent in time and money will be less, and as a result, the interpretation will be correct, data will be more understandable. Thus, over time, increasingly added value will be created. However, it is inevitable that the type of organization and its strategy and competitive advantage should also be considered. As these results are general, and they should be customized according to the organization.

This research has pursued the exploratory approach and has not followed the descriptive approach. In the future, it is possible to follow the descriptive approach with a structural equation to analyze the effects of data dimensions on an organization's readiness for implementing and analyzing BI at different maturity levels.

It is recommended to perform a case study on an organization that has implemented $\mathrm{BI}$ to examine the importance of the introduced indicators and to review the compliance of exploration results with the quantitative results of this study. In addition, in future studies, the importance of introduced indicators can be measured in terms of different maturity levels (especially technical models).

\section{REFERENCES}

[1] H. K. Bhargava, D. J. Power, and D. Sun, "Progress in web-based decision support technologies," Decision Support Systems, vol. 43, no 4, pp. 1083-1095, 2007

[2] J. Dekkers, J. Versendaal, and R. Batenburg, "Organising for business intelligence: A framework for aligning the use and development of information," BLED 2007 Proceedings, p. 15, 2007.

[3] C. Sacu and M. R. Spruit, "BIDM-The business intelligence development model," in Proc. ICEIS, 2010, vol. 1, pp. 288-293.

[4] P. Hawking, "Business intelligence excellence: A company's journey to business intelligence maturity," in Proc. the 17th Americas Conference on Information Systems, Detroit, Michigan, USA, pp. 1-9. 2011.

[5] P. Fraser, J. Moultrie, and M. Gregory, "The use of maturity models/grids as a tool in assessing product development capability," in IEEE International Proc. Engineering Management Conference, 200., vol. 1, pp. 244-249.

[6] E. Turban et al., Business Intelligence: A managerial Approach, Upper Saddle River, NJ: Pearson Prentice Hall, 2008.

[7] O. Isik, "Business intelligence success: An empirical evaluation of the role of BI capabilities and organization's decision environment," AMCIS 2009 Doctoral Consortium, 2009, p. 13.

[8] C. Howson, Successful Business Intelligence, Tata McGraw-Hill Education, 2007

[9] A. H. Anjariny and A. M. Zeki, "Development of model for assessing organizations' readiness toward successful Business Intelligence systems," in Proc. 2011 International Conference on Research and Innovation in Information Systems, 2011.

[10] N. H. Rasmussen, P. S. Goldy, and P. O. Solli, Financial Business Intelligence: Trends, Technology, Software Selection, and Implementation, John Wiley \& Sons, 2002.

[11] B. H. Wixom, H. J. Watson, A. M. Reynolds, and J. A. Hoffer, "Continental airlines continues to soar with business intelligence," Information Systems Management, vol. 25, no. 2, pp. 102-112, 2008.

[12] W. Yeoh and A. Koronios, "Critical success factors for business intelligence systems," Journal of computer information systems, vol. 50, no. 3, pp. 23-32, 2010.

[13] A. Popovic and J. Jaklic, "Benefits of business intelligence system implementation: an empirical analysis of the impact of business intelligence system maturity on information quality," in Proc. European, Mediterranean \& Middle Eastern Conference on Information Systems, Abu Dhabi, UAE, April 2010.

[14] S. Negash, "Business intelligence," The Communications of the Association for Information Systems, vol. 13, no. 1, pp. 54, 2004. 
[15] R. Y. Wang and D. M. Strong, "Beyond accuracy: What data quality means to data consumers," Journal of Management Information Systems, vol. 12, no.4, pp. 5-33, 1996.

[16] I. H. Rajterič, "Overview of business intelligence maturity models. Management," Journal of Contemporary Management Issues, vol. 15, no. 1, pp. 47-67, 2010.

[17] G. Shanks and B. Corbitt, "Understanding data quality: Social and cultural aspects, in Proc. the 10th Australasian Conference on Information Systems, vol. 785, Victoria University of Wellington, New Zealand, December 1999.

[18] N. Laranjeiro, S. N. Soydemir, and J. Bernardino, "A survey on data quality: classifying poor data," in Proc. 2015 IEEE 21 st Pacific Rim International Symposium on Dependable Computing, 2015.

[19] M. Rantala, Data Quality Analysis in Industrial Maintenance; Theory vs. Reality, 2016.

[20] D. Larson and V. Chang, "A review and future direction of agile, business intelligence, analytics and data science," International Journal of Information Management, vol. 36, no. 5, pp. 700-710. 2016.

[21] M. Najmi, M. Sepehri, and S. Hashemi, "The evaluation of Business Intelligence maturity level in Iranian banking industry," in Proc. 2010 IEEE 17Th International Conference on Industrial Engineering and Engineering Management, October 2010, pp. 466-470.

[22] S. Negash and P. Gray, Business Intelligence, Springer Berlin Heidelberg, pp. 175-193, 2008.

[23] S. Williams and N. Williams, The Profit Impact of Business Intelligence, Morgan Kaufmann, 2010.

[24] P. Darke and G. Shanks, "User viewpoint modelling: Understanding and representing user viewpoints during requirements definition," Information Systems Journal, vol. 7, no. 3, pp. 213-219, 1997.

[25] R. Kimball, The Data Warehouse Lifecycle Toolkit, John Wiley \& Sons, 2008.

[26] C. Batini and M. Scannapieco, "Data quality dimensions," Data and Information Quality, pp. 21-51, Springer International Publishing, 2016.

[27] D. M. Strong, Y. W. Lee, and R. Y. Wang, "Data quality in context," Communications of the ACM, vol. 40, no. 5, pp. 103-110, 1997.

[28] B. Burton, Results of Business Intelligence and Performance Management Maturity Survey, Gartner Inc. Research, 2007.

[29] F. Naumann and C. Rolker, Assessment Methods for Information Quality Criteria, 2000.

[30] D. Loshin, The Practitioner's Guide to Data Quality Improvement, Elsevier, 2010.

[31] S. Knight and J. M. Burn, "Developing a framework for assessing information quality on the World Wide Web," Informing Science: International Journal of an Emerging Transdiscipline, vol. 8, no. 5, pp. 159-172, 2005

[32] A. Dedeke, A Conceptual Framework for Developing Quality Measures for Information Systems, 2000.

[33] I. Caballero, M. Serrano, and M. Piattini, "A data quality in use model for big data," in Proc. International Conference on Conceptual Modeling, Springer, 2014

[34] J. Merino, I. Caballero, B. Rivas, M. Serrano, and M. Piattini, "A data quality in use model for big data," Future Generation Computer Systems, vol. 63, pp. 123-130, 2015.

[35] Z. Song, Y. Sun, J. Wan, and P. Liang, "Data quality management for service-oriented manufacturing cyber-physical systems," Computers \& Electrical Engineering, 2016.

[36] C. Cappiello, T. D. Noia, B. A. Marcu, and M. Matera, "A Quality model for linked data exploration," in Proc. International Conference on Web Engineering, pp. 397-404, Springer International Publishing, June 2016.

[37] H. Huang, B. Stvilia, C. Jörgensen, and H. W. Bass, "Prioritization of data quality dimensions and skills requirements in genome annotation work," Journal of the American Society for Information Science and Technology, vol. 63, no. 1, pp. 195-207, 2012.

[38] M. J. Eppler and D. Wittig, Conceptualizing Information Quality: A Review of Information Quality Frameworks from the Last Ten Years, in $I Q, 2000$.

[39] B. K. Kahn, D. M. Strong, and R. Y. Wang, "Information quality benchmarks: product and service performance," Communications of the ACM, vol. 45, no. 4, pp. 184-192, 2002.

[40] B. D. Klein, "User perceptions of data quality: Internet and traditional text sources," The Journal of Computer Information Systems, vol. 41, no. 4 , p. 9. 2001.

[41] X. Zhu and S. Gauch, "Incorporating quality metrics in centralized/distributed information retrieval on the world wide web," in Proc. the 23rd Annual International ACM SIGIR Conference on Research and Development in Information Retrieval, 2000, ACM.

[42] S.-T. Liaw, A. Rahimi et al., "Towards an ontology for data quality in integrated chronic disease management: A realist review of the literature," International journal of medical informatics, vol. 82, no. 1, pp. 10-24, 2013

[43] M. Janssen, H. van der Voort, and A. Wahyudi, "Factors influencing big data decision-making quality," Journal of Business Research, vol. 70, pp. 338-345, 2017

[44] L. L. Pipino, Y. W. Lee, and R. Y. Wang, "Data quality assessment," Communications of the ACM, vol. 45, no. 4, pp. 211-218, 2002.

[45] M. Tavana, D. T. Kennedy, J. Rappaport, and Y. J. Ugras, "An AHP-Delphi group decision support system applied to conflict resolution in hiring decisions," Journal of Management System, vol. 5, no. 1, pp. 49-74, 1993.

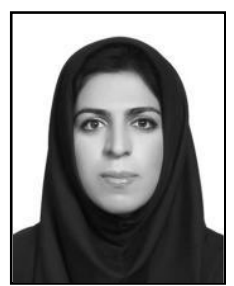

Aylin Hejazi received her B.Eng degree in computer engineering from Urmia University of Technology, Iran in 2013 and master degree in IT management from Alzahra University, Tehran, Iran in 2016. She is interested in research in the field of information systems \& technology, including business intelligence, knowledge management, and information systems.

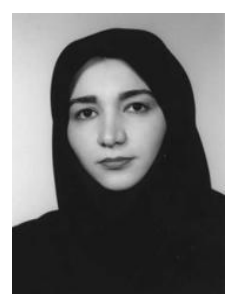

Neda Abdolvand is an associate professor in Alzahra University, and was a postdoctoral research fellow at Tarbiat Modares University. She holds her PhD and MS in information technology from Tarbiat Modares University and a post graduate certificate in information systems from Melbourne University. She is interested in research in the field of information systems \& technology, including business intelligence, and big data analytics. She has been a member of the Association for Information Systems since 2009.

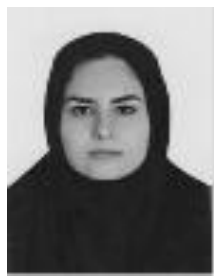

Saeedeh Rajaee Harandi holds her MA in information technology management from Alzahra University, Tehran, Iran. She is interested in research in the field of information management systems, and ICT, including business intelligence, and knowledge management. 